\title{
Duty-Ratio Feedforward for Digitally Controlled Boost PFC Converters
}

\author{
David M. Van de Sype*, Koen De Gussemé, Alex P. Van den Bossche and Jan A. Melkebeek \\ Electrical Energy Laboratory \\ Department of Electrical Energy, Systems and Automation, Ghent University \\ Sint-Pietersnieuwstraat 41, B-9000 Gent, Belgium \\ *E-mail:vdsype@lambda.rug.ac.be
}

\begin{abstract}
When a 'classical' current control scheme is applied, the input current of a boost power factor correction (PFC) converter leads the input voltage, resulting in a nonunity fundamental displacement factor and in important zerocrossing distortion in applications with a high grid frequency (e.g. $400 \mathrm{~Hz}$ power systems on commercial aircraft). To resolve this problem, a current-control scheme is proposed using duty-ratio feedforward. In this paper, the input impedance of the boost PFC converter for both the classical current-loop controller and the controller using duty-ratio feedforward are derived theoretically. A comparison reveals the advantages of the proposed control scheme: a low total-harmonic-distortion (THD) of the input current, a resistive input impedance, virtually no zero-crossing distortion and a fundamental displacement power factor close to unity. The theoretical results obtained are verified using an experimental setup of a digitally controlled boost PFC converter.
\end{abstract}

\section{INTRODUCTION}

During the last decade, there has been a large interest in power factor correction (PFC). Hence, a large number of papers tackling issues on control of PFC converters have appeared [1]-[15]. For reasons of price, the control algorithms for single-phase PFC converters, are in most cases implemented as analog circuits [1]-[7]. With the advent of fast digital signal processors (DSP) embedding control peripherals



Fig. 1. Schematic diagram of the digitally controlled boost PFC converter such as PWM generation units, AD converters, etc. (e.g. TMS320C2XX of Texas Instruments, ADMCXXX of Analog Devices, DSP56800 of Motorola), new and more complicated control algorithms become feasible. For the near future, as the ratio price/performance of DSP's is expected to decrease further, there is a fair chance that the analog control circuits will be abandoned in favor of digital implementations. This tendency can also be illustrated by the recent interest in digital control of PFC converters [8]-[15].

When a 'classical' current control scheme is applied, the input current of a boost PFC converter leads the input voltage, resulting in a non-unity fundamental displacement factor and in important zero-crossing distortion in applications with a high grid frequency (e.g. $400 \mathrm{~Hz}$ power systems on commercial aircraft) [7]. To resolve this problem, a current-control scheme is proposed using duty-ratio feedforward. This currentloop controller was already shown in [11], but as this paper focussed on the design of a fast voltage-loop controller, the current-loop controller was only mentioned briefly. The extra circuit complexity and cost related to an analog implementation of the current-loop controller in [11] may not always counterbalance the advantages of the control scheme. However, using a digital controller, the increased controller complexity results in only a few extra lines of programming code. Consequently, the current-loop controller using dutyratio feedforward is revised. In this paper, the input impedance of the boost PFC converter for both the classical currentloop controller and the controller using duty-ratio feedforward are derived theoretically. A comparison of the two controllers reveals the advantages of the proposed control scheme: a low total-harmonic-distortion (THD) of the input current, a resistive input impedance, almost no zero-crossing distortion and a fundamental displacement factor close to unity. The theoretical results obtained are verified using an experimental setup of a digitally controlled boost PFC converter.

Fig. 1 shows the circuit diagram employed. For the purpose of digital control, the analog control variables (the input current $i_{L}$, the input voltage $v_{i n}$ and the output voltage $v_{o}$ ) must be converted into digital quantities by the ADC converter. The process of sensing the control variable, amplifying the sensor output to the appropriate range and the analog-to-digital conversion, can be represented by a division of the analog control variables by their respective reference values $\left(I_{L}^{r e f}\right.$, 

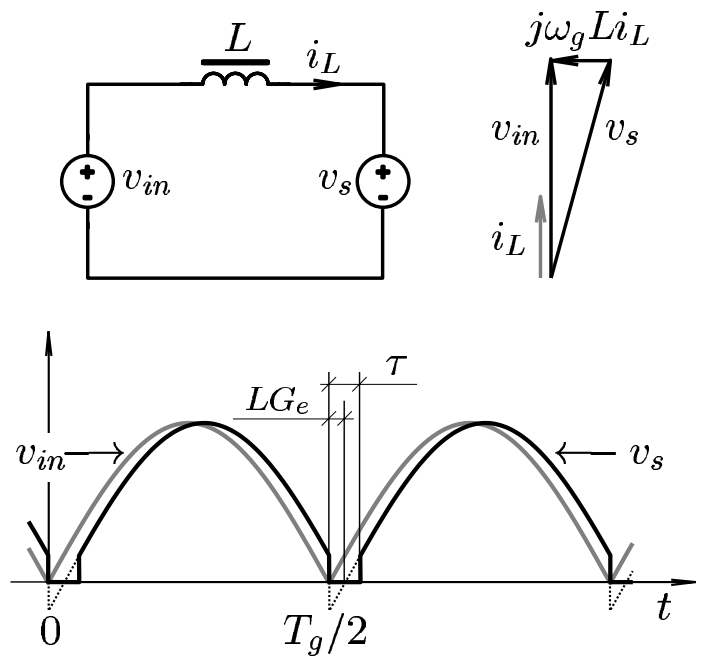

Fig. 2. A model for the operation of a boost PFC converter and key operating waveforms

$V_{i n}^{r e f}$ and $\left.V_{o}^{r e f}\right)$. The obtained digital dimensionless quantities $i_{L, n}, v_{i n, n}$ and $v_{o, n}$ are used by the digital controller to act on the duty-ratio $d_{n}$ of the switch $S$. The objective of the digital controller is to maintain a constant output voltage while guaranteeing low harmonic content of the input current.

\section{The Switch Voltage}

Assuming a perfect sinusoidal grid voltage, the voltage at the input of the boost converter is a rectified sinusoid $v_{\text {in }}$ with angular frequency $\omega_{g}$ (Fig. 2). Considering only half a grid period $\left[0, T_{g} / 2\right]$ (with $T_{g}$ the grid period), the input voltage can be expressed as

$$
v_{\text {in }}(t)=\widehat{V}_{i n} \sin \left(\omega_{g} t\right) .
$$

The average switch voltage $v_{s}$ (of the switch $S$ in Fig. 1) required to obtain an input current $i_{L}$ identical in waveshape to, and in phase with the input voltage $v_{i n}$ in the time interval $\left[0, T_{g} / 2\right]$, can be expressed (Fig. 2) using complex vector notation

$$
v_{s}=v_{i n}-j \omega_{g} L i_{L}=v_{i n}\left(1-j \omega_{g} L G_{e}\right),
$$

with $G_{e}=\frac{i_{L}}{v_{i n}}$, the emulated conductance at the input of the boost PFC converter. By taking into account that $\omega_{g} L G_{e} \ll 1$, the average switch voltage becomes

$$
v_{s}(t)=\operatorname{sign}\left(\sin \left(\omega_{g} t\right)\right) \widehat{V}_{s} \sin \left(\omega_{g}\left(t-L G_{e}\right)\right),
$$

with

$$
\widehat{V}_{s}=\widehat{V}_{i n} \sqrt{1+\left(\omega_{g} L G_{e}\right)^{2}} \approx \widehat{V}_{i n} .
$$

Hence, the average switch voltage should be as indicated by the dotted line in Fig. 2: a 'rectified' sinusoid delayed over a time $L G_{e}$, with a magnitude slightly larger than the input voltage and with a discontinuity at the zero-crossing of the grid voltage. For a boost converter operating in continuous conduction mode the average switch voltage is

$$
v_{s}=(1-d) v_{o},
$$

with $d$ the duty-ratio of the switch $S$ and $v_{o}$ the output voltage. As the output voltage of a boost PFC converter is always positive and the duty-ratio is limited between 0 and 1 , the average switch voltage must always be positive. However, according to (3) and Fig. 2 (dotted line), the average switch voltage should be negative at the zero-crossing of the grid voltage. This discrepancy is the cause for the occurrence of the so-called cusp distortion. After all, as the switch voltage can't become negative, the switch voltage remains zero until the inductor current is back on track (time $\tau$ Fig. 2). The time $\tau$ necessary to get the inductor current back on track with a closed switch $S$, can be calculated using the differential equation of the boost inductor $L$

$$
L \frac{\mathrm{d} i_{L}}{\mathrm{~d} t}=v_{i n} .
$$

By using (1), the differential equation (6) can be integrated over a time $\tau$

$$
i_{L}(\tau)-i_{L}(0)=\frac{\widehat{V}_{i n}}{\omega_{g} L}\left(1-\cos \left(\omega_{g} \tau\right)\right)
$$

The desired input current is $G_{e} v_{i n}$ with $v_{i n}$ given by (1). Expressing that the switch voltage remains zero until the input current is back on track yields

$$
G_{e} \widehat{V}_{i n} \sin \left(\omega_{g} \tau\right)=\frac{\widehat{V}_{i n}}{\omega_{g} L}\left(1-\cos \left(\omega_{g} \tau\right)\right) .
$$

For small $\omega_{g} \tau$ the goniometric functions of (8) can be approximated by their second order polynomials

$$
G_{e} \omega_{g} \tau=\frac{1}{\omega_{g} L}\left(1-\left(1-\frac{\left(\omega_{g} \tau\right)^{2}}{2}\right)\right),
$$

or

$$
\tau=2 L G_{e} .
$$

Hence, the average switch voltage should remain zero during a time $2 L G_{e}$ after the zero-crossing of the grid voltage, before it can adopt its normal value (3) again. The resulting average switch voltage is indicated as a black line in Fig. 2.

\section{Duty-RATio FEEDFORWARD}

\section{A. The Current Controller Using Duty-Ratio Feedforward}

As a resistive input is a desirable feature for a PFC converter, the task of the current-loop compensator consists of forcing the input current to be identical in waveshape to the input voltage. Hence, the current-loop compensator should reconstruct as accurately as possible the average switch waveform $v_{s}$ of Fig. 2 (black line) from the input current error. As the time delay $L G_{e}$ between the input voltage $v_{i n}$ and the average switch voltage $v_{s}$ is usually very small (in our case $<20 \mu \mathrm{s}$ ), both waveforms are almost identical. Hence, if the input voltage is added to the output of the current-loop compensator, the latter only has to compensate for the small difference between $v_{i n}$ and $v_{s}$ instead of compensating for $v_{s}$ entirely. This results in a smaller input current error and as 


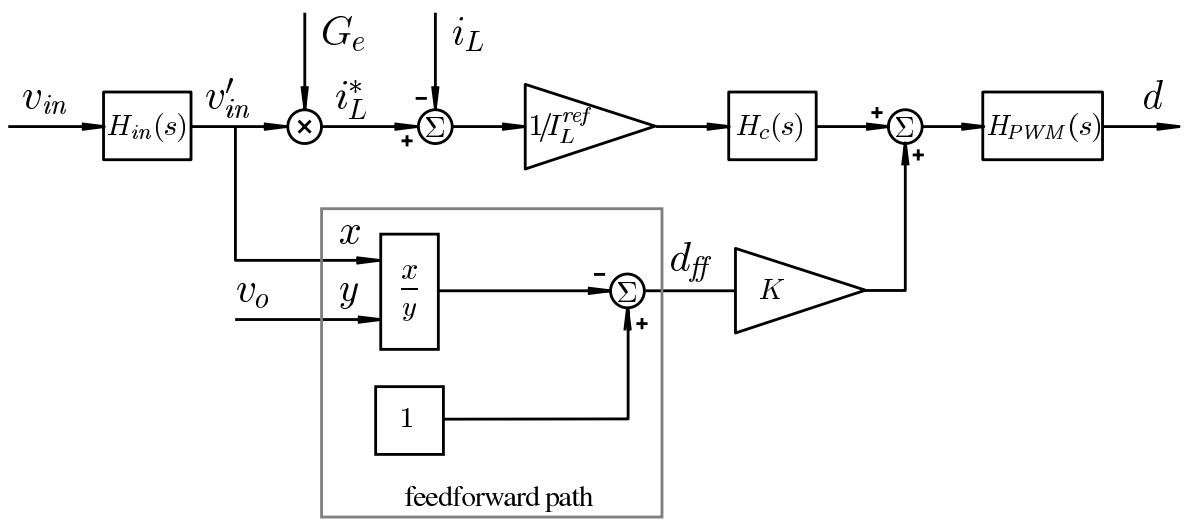

Fig. 3. The current loop controller

a consequence in a better tracking of the commanded input current. Hence, the forwarded switch voltage $v_{s, f f}$ becomes

$$
v_{s, f f}=v_{i n} .
$$

By considering (5) for a boost converter operating in continuous conduction mode, the forwarded duty-ratio $d_{f f}$ is

$$
d_{f f}=1-\frac{v_{i n}}{v_{o}} .
$$

The resulting control scheme is depicted in Fig. 3. The controller consists of a 'regular' path and a feedforward path. For the regular path, the inductor current error, the difference between the inductor current $i_{L}$ and the commanded inductor current $i_{L}^{*}$, is scaled (division by $I_{L}^{\text {ref }}$, see also Fig. 1) and supplied to the current compensator $H_{c}(s)$. The output of the compensator is passed on to the pulse-width modulator (Fig. 1) with as transfer function $H_{P W M}(s)$. The commanded input current $i_{L}^{*}$ is acquired by multiplying the filtered input voltage $v_{i n}^{\prime}$ (the input voltage filtered by a low-pass filter) with the equivalent input conductance $G_{e}$. This commonly employed current control scheme is extended with a dutyratio feedforward path implementing (12). The output of the feedforward path $d_{f f}$ is multiplied with a constant $K$ that is allowed to be either 0 or 1 , corresponding to the current-loop controller without or with duty-ratio feedforward, respectively.

\section{B. The Input Impedance}

If the output voltage $v_{o}$ of the boost PFC converter is not constant, the feedforward path (12) introduces a non-linearity in the control path. To calculate the input impedance of the boost PFC converter with a feedforward current control loop, linearization is required. Using capitals for steady-state values and hatted small letters for small excursions from steady-state, the linearized version of the non-linear feedforward path (12) becomes

$$
\widehat{d}_{f f}(s)=\frac{V_{i n}^{\prime}}{V_{o}^{2}} \widehat{v}_{o}(s)-\frac{1}{V_{o}} \widehat{v}_{i n}^{\prime} .
$$

Taking into account that $V_{i n}^{\prime} \approx V_{i n}$ and $\widehat{v}_{i n}^{\prime}(s)=H_{\text {in }}(s) \widehat{v}_{i n}(s)$, allows to derive the small-signal transfer function of the current loop controller (Fig. 3)

$$
\begin{gathered}
\widehat{d}(s)=-\frac{H_{P W M}(s) H_{c}(s)}{I_{L}^{r e f}} \widehat{\imath}_{L}(s)+K \frac{V_{i n}}{V_{o}^{2}} H_{P W M}(s) \widehat{v}_{o}+ \\
{\left[\left(\frac{G_{e} H_{c}(s) H_{i n}(s)}{I_{L}^{\text {ref }}}-K \frac{H_{i n}(s)}{V_{o}}\right) H_{P W M}(s)\right] \widehat{v}_{i n}(s) .}
\end{gathered}
$$

The response of the boost converter can be expressed as

$$
\widehat{\imath}_{L}(s)=\frac{1}{s L} \widehat{v}_{i n}(s)+\frac{V_{o}}{s L} \widehat{d}(s)-\frac{(1-D)}{s L} \widehat{v}_{o} .
$$

By eliminating the duty-ratio $\widehat{d}$ in (14) and (15), the input current of the converter becomes

$$
\begin{gathered}
\widehat{\imath}_{L}(s)\left(s L+\frac{V_{o}}{I_{L}^{\text {ref }}} H_{c}(s) H_{P W M}(s)\right)= \\
\widehat{v}_{o}(s)(1-D)\left(K H_{P W M}(s)-1\right)+ \\
\widehat{v}_{i n}(s)\left[\left(1-K H_{i n}(s) H_{P W M}(s)\right)+\right. \\
\left.\frac{V_{o} G_{e}}{I_{L}^{\text {ref }}} H_{c}(s) H_{i n}(s) H_{P W M}(s)\right] .
\end{gathered}
$$

Hence, the small-signal input impedance is

$$
\begin{aligned}
& \widehat{z}_{i n}(s)=\frac{\widehat{v}_{i n}(s)}{\widehat{\imath}_{L}(s)}= \\
& \frac{s L+\frac{V_{o}}{I_{L}^{\text {ref }}} H_{c}(s) H_{P W M}(s)}{\left(1-K H_{\text {in }}(s) H_{P W M}(s)\right)+\frac{V_{o} G_{e}}{I_{L}^{\text {ref }}} H_{c}(s) H_{\text {in }}(s) H_{P W M}(s)} .
\end{aligned}
$$

To avoid any influence of the unknown grid impedance on the basic operation of the boost PFC converter it is necessary to insert the input capacitor $C_{i n}$ (Fig. 1). After all, only when the impedance of the input capacitor at the switching frequency is much smaller than that of the inductor $L$, the grid impedance will have a negligible influence on the inductor-current-ripple waveform, which allows to derive (15). Consequently, the input impedance $\widehat{z}_{i n}$ can't be considered separately from the impedance of the capacitor $C_{i n}$. Therefore, the total input impedance $\widehat{z}_{\text {tot }}$ is defined as

$$
\widehat{z}_{\text {tot }}(s)=\left(\widehat{z}_{i n}^{-1}(s)+s C_{i n}\right)^{-1} .
$$



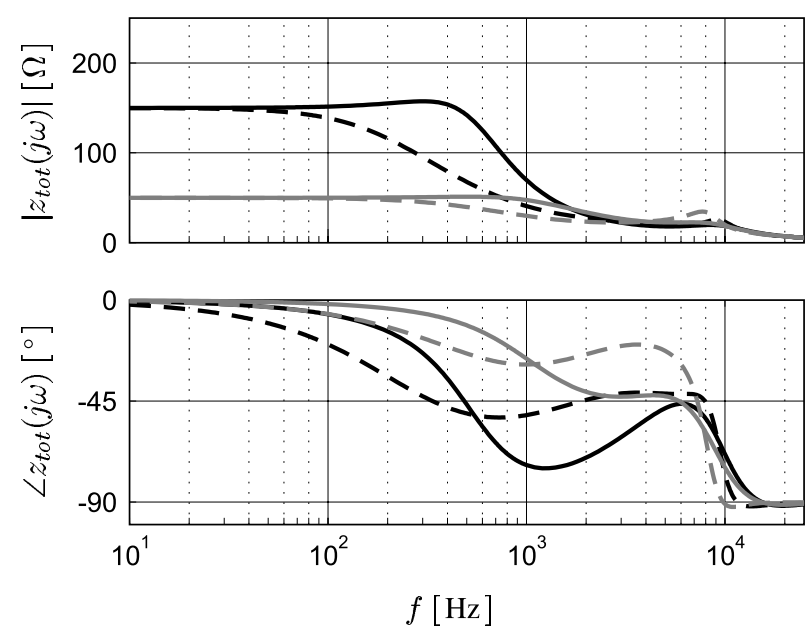

Fig. 4. The total input impedance $\widehat{z}_{t o t}$ of the boost PFC converter (dashed lines: without feedforward, full lines: with feedforward, black lines: $G_{e}=$ $(150 \Omega)^{-1}$, gray lines: $\left.G_{e}=(50 \Omega)^{-1}\right)$

\section{The Different Transfer Functions for a Digitally Controlled Boost PFC Converter}

To obtain quantitative values for the input impedances (17) and (18) the transfer functions of the different blocks in the digital controller of the boost PFC converter are required. As the different transfer functions were derived in [14], [15] and [16], the results are only shortly listed in this paper.

- For reasons of stability a low-pass filter is inserted in signal chain of the input voltage $v_{\text {in }}$ [2]. As low-pass filter a first-order analog filter was inserted before the ADC.

$$
H_{i n}(s)=\frac{1}{1+s \tau_{f}} .
$$

- Though the current compensator is implemented digitally, the transfer function of its continuous equivalent is used with good accuracy

$$
H_{c}(s)=K_{P I}\left(1+\frac{1}{s \tau_{P I}}\right) .
$$

- As modulator, a uniformly sampled symmetric-on-time modulator is used. In [16] a frequency domain model for this modulator was derived. Taking into account an extra delay of half a switching period $T / 2$ for the calculation of the control output, the modulator response can be derived (in the frequency domain)

$$
H_{P W M}(j \omega)=\underbrace{\cos \left(\frac{\omega D T}{2}\right) e^{-j \frac{\omega T}{2}}}_{\text {modulator }} \cdot \underbrace{e^{-j \frac{\omega T}{2}}}_{\text {delay }}
$$

\section{Discussion of Results}

The input current $\widehat{\imath}_{L}$ (16) of a boost PFC converter with a current-control loop is determined by two external inputs: the input voltage $\widehat{v}_{i n}$ and the output voltage $\widehat{v}_{o}$. While the response to the input voltage results in an input impedance (17), the reaction of the input current to a change in the output voltage
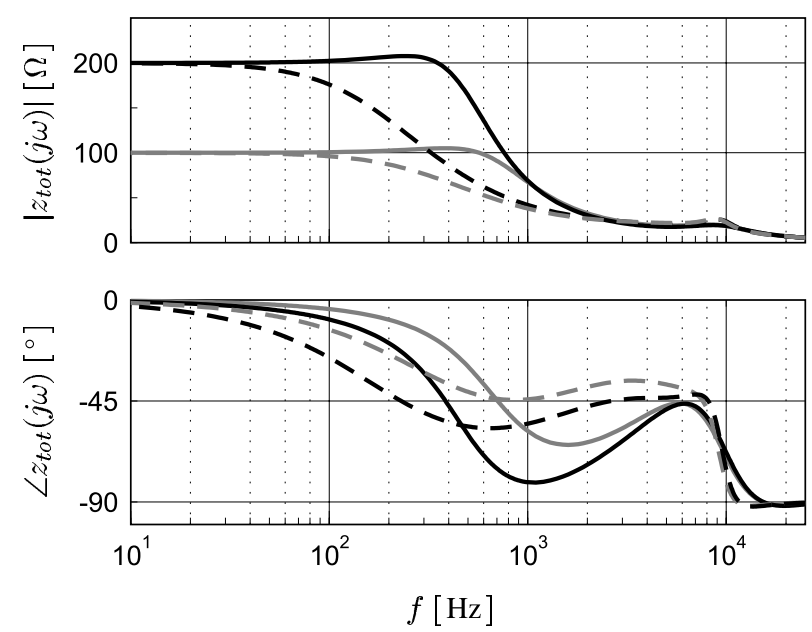

Fig. 5. The total input impedance $\widehat{z}_{\text {tot }}$ of the boost PFC converter (dashed lines: without feedforward, full lines: with feedforward, black lines: $G_{e}=$ $(200 \Omega)^{-1}$, gray lines: $\left.G_{e}=(100 \Omega)^{-1}\right)$

is considered to be a disturbance. The transfer function from this disturbance to the input current is

$$
\frac{\widehat{\imath}_{L}}{\widehat{v}_{o}}=\frac{(1-D)\left(K H_{P W M}(s)-1\right)}{\left(s L+\frac{V_{o}}{I_{L}^{\text {ref }}} H_{c}(s) H_{P W M}(s)\right)} .
$$

When no feedforward is applied $(K=0)$, disturbance rejection is achieved by making the denominator of (22) large i.e. by choosing a compensator $H_{c}(s)$ with a large gain. As a PIcompensator (20) has a high gain for 'low' frequencies and since the output voltage is in most cases a slow signal due to the large output capacitor $C_{o}$ (Fig. 1), sufficient disturbance rejection is achieved. However, when feedforward $(K=1)$ is applied, the numerator of (22) is virtually zero for 'low' frequencies. Hence, in the case of feedforward, disturbance rejection doesn't rely on whether the compensator has a high gain or not.

For $0<\omega<\frac{1}{\tau_{P I}}, \tau_{P I} \gg \tau_{f}$ and $\tau_{P I} \gg T$, the input impedance (17) can be approximated by ( $\omega L$ is small)

$$
\widehat{z}_{i n}(j \omega) \approx \frac{\frac{V_{o}}{I_{L}^{r e f}} \cdot \frac{K_{P I}}{j \omega \tau_{P I}}}{(1-K)+G_{e} \cdot \frac{V_{o}}{I_{L}^{\text {ref }}} \cdot \frac{K_{P I}}{j \omega \tau_{P I}}},
$$

or

$$
\widehat{z}_{i n}(j \omega) \approx \frac{\alpha}{j \omega(1-K)+G_{e} \alpha}
$$

with

$$
\alpha=\frac{V_{o}}{I_{L}^{r e f}} \cdot \frac{K_{P I}}{\tau_{P I}} .
$$

Using a regular control scheme $(K=0)$, the input impedance of the boost PFC converter (24) has a positive real part and a negative imaginary part, indicating resistive-capacitive behavior. As a result the input current of the boost PFC converter using a regular control scheme will lead the input voltage, causing a non-unity fundamental displacement factor. 

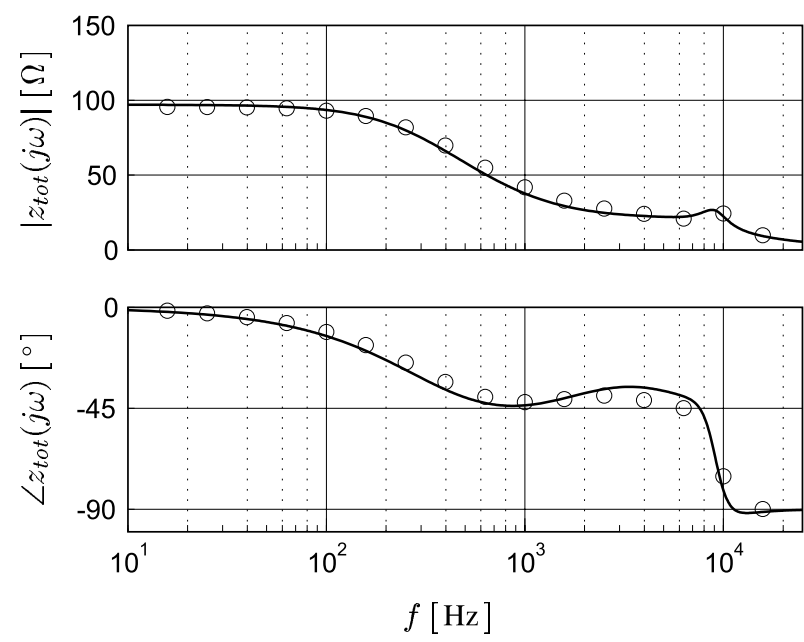

Fig. 6. The total input impedance $\widehat{z}_{\text {tot }}$ without feedforward for $G_{e}=(97 \Omega)^{-1}$, theory (solid line) versus measurement (circles)
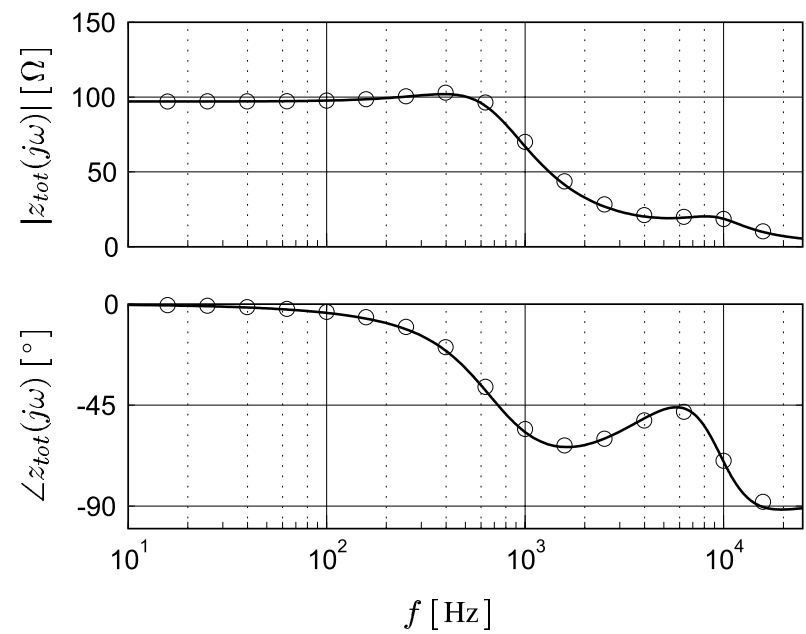

Fig. 7. The total input impedance $\widehat{z}_{t o t}$ with feedforward for $G_{e}=(97 \Omega)^{-1}$, theory (solid line) versus measurement (circles)

Moreover, the input impedance $\widehat{z}_{i n}$ will only approximate its desired value $\left(G_{e}\right)^{-1}$ as long as $G_{e} \alpha \gg \omega$. Hence, at light load and for increasing frequency the input impedance will not accurately represent the desired input impedance. However, if the control scheme with feedforward is applied $(K=1)$, the input impedance (24) behaves resistive and is equal to its desired value $\left(G_{e}\right)^{-1}$ independent of frequency or load conditions. Consequently, the boost PFC converter using a feedforward controller will have a fundamental displacement factor close to unity and will behave resistive over a wide frequency range, guaranteeing superior behavior of the boost PFC converter.

Figs. 4 and 5 show frequency plots of the total input impedance $\widehat{z}_{\text {tot }}$ (18) of the boost PFC converter for different values of the desired input conductance $G_{e}$. The different parameters required to calculate (17) and (18) were chosen similar to those of the experimental setup (see section IV). As the total input impedance $\widehat{z}_{\text {tot }}$ also includes the input capacitor
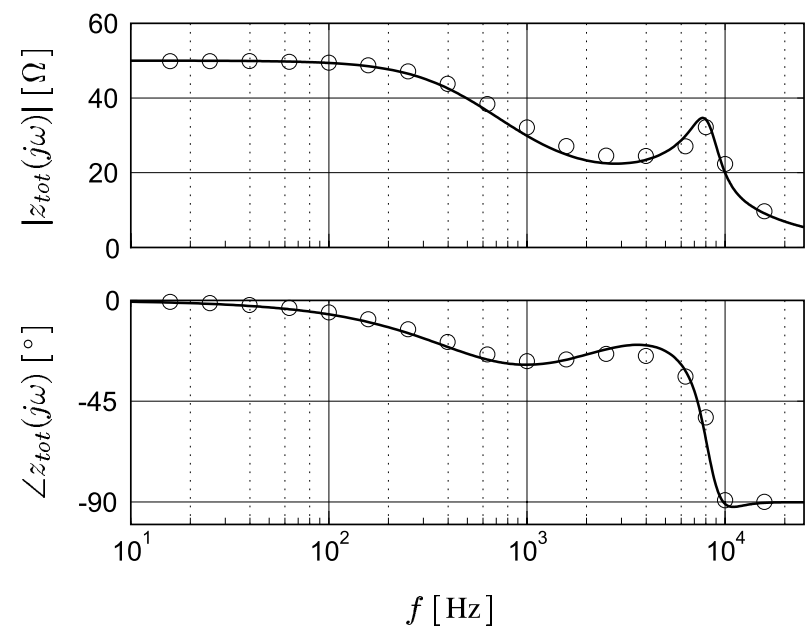

Fig. 8. The total input impedance $\widehat{z}_{\text {tot }}$ without feedforward for $G_{e}=(50 \Omega)^{-1}$, theory (solid line) versus measurement (circles)
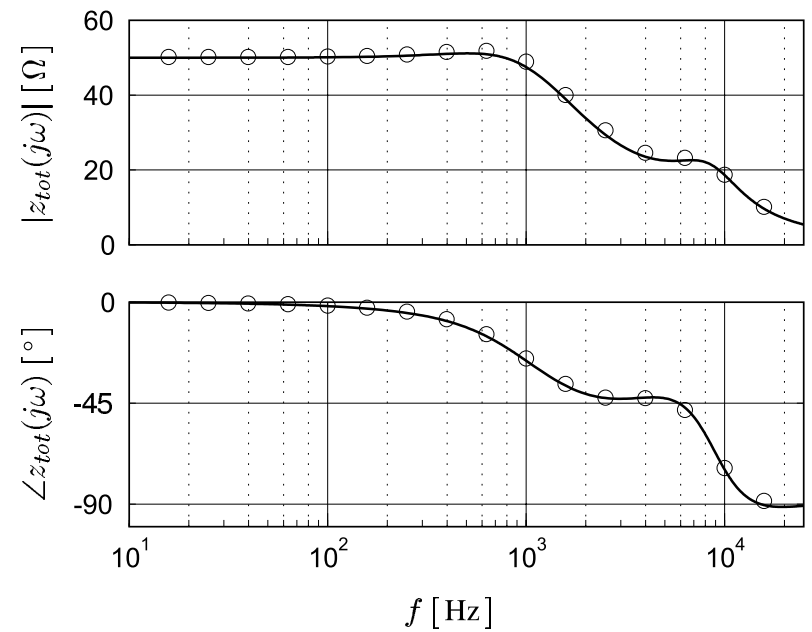

Fig. 9. The total input impedance $\widehat{z}_{t o t}$ with feedforward for $G_{e}=(50 \Omega)^{-1}$, theory (solid line) versus measurement (circles)

$C_{i n}(18)$, the total input impedance always behaves capacitive as opposed to the input impedance $\widehat{z}_{i n}$. Nevertheless, the advantages of the current-control loop using feedforward over the one without feedforward is clearly visible. When feedforward is applied, the magnitude of the total input impedance remains nearly equal to the desired input impedance $\left(G_{e}\right)^{-1}$ up to the frequency where the impedance of the input capacitor $C_{i n}$ becomes significant (Figs. 4 and 5). For the control scheme without feedforward, the magnitude of the total input impedance already sets appreciably at low frequencies. This effect is even more pronounced for high values of the desired input impedance $\left(G_{e}\right)^{-1}$ (see explanation previous paragraph concerning (24)). Moreover, the imaginary part of the total input impedance of the converter applying feedforward is much smaller in the low frequency range than for the converter without feedforward (Figs. 4 and 5). Hence, application of feedforward guarantees a resistive behavior of the boost PFC converter for low frequencies and a fundamental displacement 


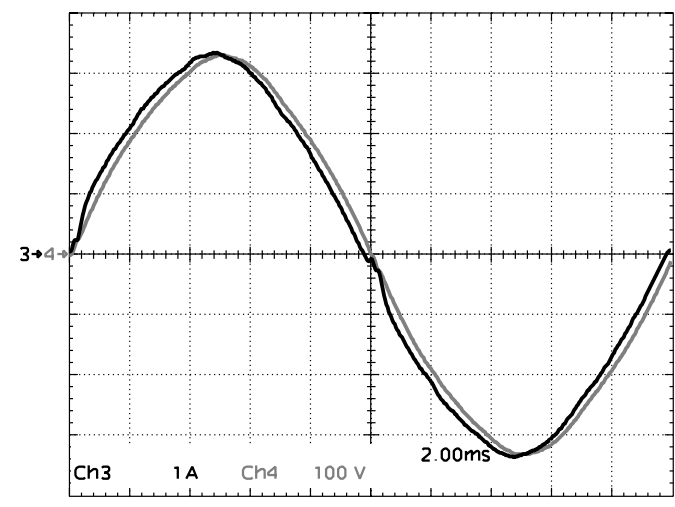

Fig. 10. Grid current and grid voltage at $50 \mathrm{~Hz}$ of the PFC converter using the current-control loop without feedforward with $G_{e}=(100 \Omega)^{-1}$ (input voltage: gray line, input current: black line)

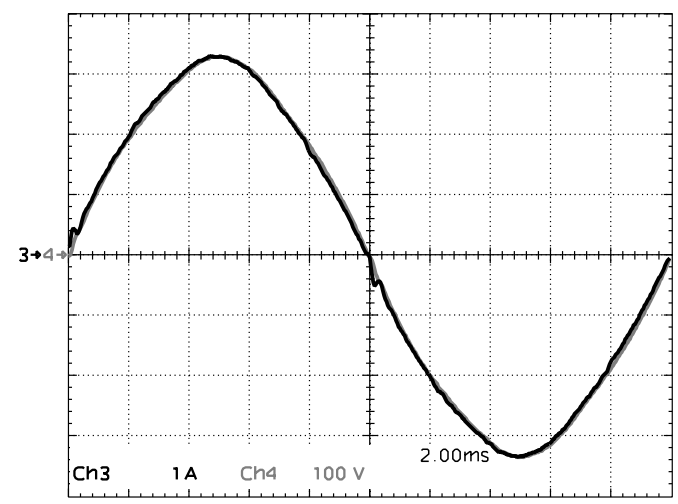

Fig. 11. Grid current and grid voltage at $50 \mathrm{~Hz}$ of the PFC converter using the current-control loop with feedforward with $G_{e}=(100 \Omega)^{-1}$ (input voltage: gray line, input current: black line)

factor close to unity.

\section{EXPERIMENTAL RESULTS}

The two current controllers were compared by using an experimental setup. The control for the boost PFC converter was implemented using the ADMC401 of Analog Devices. The switches of the boost rectifier are $S$ MOSFET SPP20N60S5 and $D$ diode RURP3060. The passive components used are $L=1 \mathrm{mH}, C_{i n}=1.2 \mu \mathrm{F}$ and $C=470 \mu \mathrm{F}$. The converter switches at $50 \mathrm{kHz}$, supplies $400 \mathrm{~V} \mathrm{DC}$ at the output and is rated at $1 \mathrm{~kW}$ output power for an input voltage range of $190 \mathrm{~V}-264 \mathrm{~V}$ AC. The parameters of the current controller are $I_{L}^{r e f}=10.45 \mathrm{~A}, K_{P I}=1.1$ and $\tau_{P I}=120 \mu \mathrm{s}$. For the control scheme without feedforward insertion of a low-pass filter in the signal path of the input voltage (19) with $\tau_{f}=22 \mathrm{~ns}$ is required for reasons of stability [2]. Conversely, when feedforward is used, no filtering of the input voltage is required to obtain stability or $\tau_{f}=0$ s.

To test the total input impedance, a linear amplifier was used to supply the boost PFC converter with a small sinusoid superposed on a DC voltage of $230 \mathrm{~V}$. With this DC voltage the average duty-ratio of the boost PFC converter is $D=(1-$ $230 \mathrm{~V} / 400 \mathrm{~V})=0.425$. The experimentally obtained values of

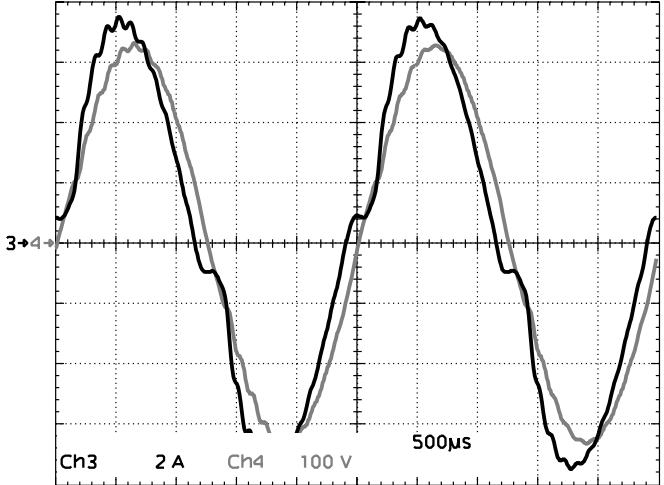

Fig. 12. Grid current and grid voltage at $400 \mathrm{~Hz}$ of the PFC converter using the current-control loop with $G_{e}=(50 \Omega)^{-1}$ without feedforward (input voltage: gray line, input current: black line)

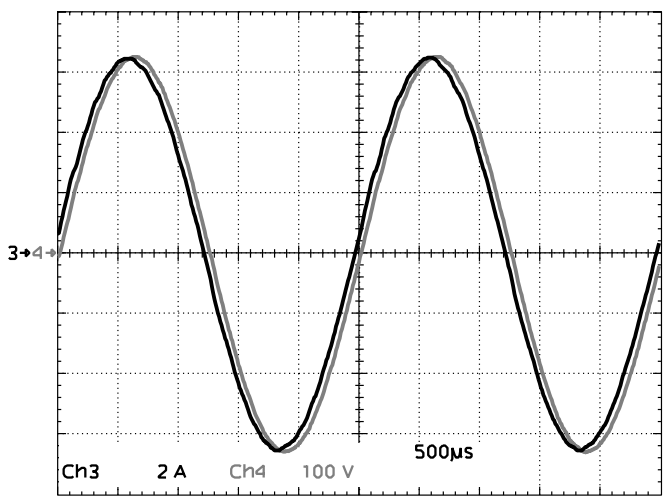

Fig. 13. Grid current and grid voltage at $400 \mathrm{~Hz}$ of the PFC converter using the current-control loop with feedforward with $G_{e}=(50 \Omega)^{-1}$ (input voltage: gray line, input current: black line)

the total input impedance of the boost PFC converter using a control circuit with or without feedforward are shown as circles in Figs. 7, 9 and Figs. 6, 8, respectively. The theoretical waveforms (compare with Figs. 4 and 5) were added as a solid line for comparison. The good agreement between the experimental results and the theoretical waveforms in Figs. 69 demonstrates the validity of the theoretical approach.

The grid waveforms obtained with the PFC converter using the current control scheme without and with feedforward are shown in Fig. 10 and Fig. 11, respectively. The input current clearly leads the input voltage when no feedforward is applied (Fig. 10), indicating that the input impedance of the converter behaves capacitive. When feedforward is used, the waveshape of the input current almost perfectly coincides with the waveshape of the input voltage (Fig. 11, note that the voltage waveform is hardly noticeable behind the input current). This shows that applying feedforward for a boost PFC converter allows to obtain a higher fundamental displacement factor.

Not only the fundamental displacement factor is ameliorated by using feedforward, but also the THD of the input current is reduced and the zero-crossing distortion problem for high grid frequencies is alleviated. These effects are demonstrated 
in Figs. 12 and 13 by connecting the boost PFC converter to a $400 \mathrm{~Hz}$ grid. When no feedforward is used, the input current waveform is severely distorted (Fig. 12). This zero-crossing distortion is caused by the leading phase of the inductor current relative to the input voltage (see section III-D) and by the low damping factor in the input-current-control loop (both effects were explained in [7]). However, when feedforward is applied, the leading phase of the input current is mainly caused by the external input capacitor $C_{i n}$ and not by the inductor current $i_{L}$. As a result, the input current of the boost PFC converter using feedforward remains a high quality waveform with a low THD and virtually no zero-crossing distortion when connected to a high frequency grid (Fig. 13).

\section{CONCLUSION}

The current-control loop commonly used for boost PFC converters operated in continuous conduction mode causes a leading phase of the inductor current relative to the input voltage. This effect results in a non-unity fundamental displacement factor and in important zero-crossing distortion in applications with a high grid frequency. To improve the behavior of the boost PFC converter a current-control scheme using feedforward of the duty-ratio is proposed. To compare both the 'classical' control scheme and the control scheme using feedforward, the input impedance of the boost PFC converter is calculated in both cases. The theoretical analysis shows that the input impedance of the converter using feedforward remains resistive with an almost constant magnitude over a wide frequency range. Hence, the main features of the converter using a current-control loop with duty-ratio feedforward are: a low THD of the input current, resistive input impedance, virtually no zero-crossing distortion and a fundamental displacement factor close to unity.

\section{REFERENCES}

[1] D. Maksimović, Y. Jang, and R.W. Erickson, "Nonlinear-carrier control for high-power-factor boost rectifiers," IEEE Trans. Power Electr., Vol. 11, No. 4, July 1996, pp. 578-584.

[2] G. Spiazzi, and J.A. Pomilio, "Interaction between EMI filter and power factor preregulators with average current control: analysis and design considerations," IEEE Trans. Ind. Electr., Vol. 46, No. 3, June 1999, pp. $577-584$.
[3] S. Ben-Yaakov, and I. Zeltser, "The dynamics of a PWM boost converter with resistive input," IEEE Trans. Ind. Electr., Vol. 46, No. 3, June 1999. pp. 613-619.

[4] J. Rajagopalan, F.C. Lee, and P. Nora, "A general technique for derivation of average current mode control laws for single-phase power-factorcorrection circuits without input voltage sensing," IEEE Trans. Power Electr., Vol. 14, No. 4, July 1999, pp. 663-672.

[5] Z. Lai, and K.M. Smedley, "A family of continuous-conduction-mode power-factor-correction controllers based on the general pulse-width modulator," IEEE Trans. Power Electr., Vol. 13, No. 3, May 1998, pp. 501-510.

[6] M.O. Eissa, S.B. Leeb, G.C. Verghese, and A.M. Stanković, "Fast controller for a unity-power-factor PWM rectifier," IEEE Trans. Power Electr., Vol. 11, No. 1, Jan. 1996, pp. 1-6.

[7] J. Sun, "Demystifying zero-crossing distortion in single-phase PFC converters," Proc. IEEE Power Electr. Spec. Conf. (PESC2002), June 23 27, 2002, Cairns, Australia, vol. 3, pp. 1109-1114.

[8] J. Zhou, Z. Lu, Z. Lin, Y. Ren, Z. Qian, and Y. Wang, "Novel sampling algorithm for DSP Controlled 2kW PFC Converter," IEEE Trans. Power Electr., Vol. 16, No. 2, March 2001, pp. 217-222. pp.185-192.

[9] H.S.H. Chung, E.P.W. Tam, S.Y.R. Hui, "Development of a fuzzy logic controller for boost rectifier with active power factor correction," Proc. IEEE Power Electr. Spec. Conf., PESC 1999, pp. 149-154.

[10] S. Buso, P. Mattavelli, L. Rossetto, and G. Spiazzi, "Simple digital control improving dynamic performance of power factor preregulators," IEEE Trans. Power Electr., Vol. 13, No. 5, Sept. 1998, pp. 814-823.

[11] S. Wall, and R. Jackson, "Fast controller design for single-phase powerfactor correction systems," IEEE Trans. Ind. Electr., Vol. 44, No. 5, Oct. 1997, pp. 654-660.

[12] A.H. Mitwalli, S.B. Leeb, G.C. Verghese, and V.J. Thottuvelil, "An adaptive digital controller for a unity power factor converter," IEEE Trans. Power Electr., Vol. 11, No. 2, March 1996, pp. 374-382.

[13] S. Sivakumar, K. Natarajan, and R. Gudelewicz, "Control of power factor controlling boost converter without instantaneous measurement of input current," IEEE Trans. Power Electr., Vol. 10, No. 4, July 1995, pp. 435445.

[14] K. De Gussemé, D.M. Van de Sype, and J.A. Melkebeek, "Design issues for digital control of boost power factor correction converters," Proc. IEEE Symp. on Ind. Electr. (ISIE2002), July 8-11, 2002, L'Aquila, Italy, vol. 3, pp. 731-736.

[15] D.M. Van de Sype, K. De Gussemé, A.P. Van den Bossche, and J.A. Melkebeek, "A sampling algorithm for digitally controlled boost PFC converters," Proc. IEEE Power Electr. Spec. Conf. (PESC2002), June 23-27, 2002, Cairns, Australia, vol. 4, pp. 1693-1698.

[16] D.M. Van de Sype, K. De Gussemé, and J.A. Melkebeek, "Frequency domain analysis of digital pulse-width modulators," Proc. Int. Conf. on Mod. and Sim. of Electr. Mach., Conv. and Syst. (Electrimacs2002), Aug. 18-21, 2002, Montréal, Canada, on cd-rom. 